\title{
Substantia Nigra Hyperechogenicity for the Early Detection of Parkinson's Disease
}

Rezzak Yilmaz ${ }^{1^{*}}$ and Daniela Berg ${ }^{1,2}$

${ }^{1}$ Department of Neurology, Christian-Albrechts-University of Kiel, Germany

${ }^{2}$ Department of Neurodegeneration, Hertie Institute for Clinical Brain Research, University of Tuebingen, Germany

"Corresponding author: Rezzak Yilmaz, Department of Neurology, Christian-Albrechts-University of Kiel, Arnold-Heller-Str. 3, 24105, Kiel, Germany, Tel: +4943150023849; Fax: +4943150023804; E-mail: Rezzak.Yilmaz@uksh.de

Received date: March 28, 2017; Accepted date: April 26, 2017; Published date: April 29, 2017

Copyright: (C) 2017 Yilmaz R, et al. This is an open-access article distributed under the terms of the Creative Commons Attribution License, which permits unrestricted use, distribution, and reproduction in any medium, provided the original author and source are credited.

\section{Abstract}

Hyperechogenicity of the substantia nigra $(\mathrm{SN}+)$ is an imaging marker which may be detected in individuals at risk for Parkinson's disease (PD). In this short commentary, the evidence of $\mathrm{SN}+$ as a risk marker for PD including its predictive value, stability and the limitations in assessment are discussed based on the current literature.

\begin{abstract}
Keywords: Transcranial sonography; Substantia nigra hyperechogenicity; Parkinson's disease

\section{Introduction}

Since the first publication by Becker and colleagues in 1995 [1], substantia nigra hyperechogenicity $(\mathrm{SN}+)$ detected by the transcranial B-mode sonography (TCS) has gained increasing popularity for clinical application and as a topic of research. Supported by numerous studies of different groups, $\mathrm{SN}+$ has meanwhile been established as a reliable marker for PD diagnosis with a sensitivity of $85-90 \%$ and a specificity of $77-86 \%$ [2-4]. Besides the interest in $\mathrm{SN}+$ as diagnostic marker, it has attracted considerable attention in the discussion of markers that may predict the risk of individuals for developing clinical $\mathrm{PD}$ [5]. In this short communication, we discuss the role of $\mathrm{SN}+$ as a risk marker for PD.
\end{abstract}

\section{Discussion}

\section{Early detection of Parkinson's disease}

Unfortunately, PD can only be diagnosed after at least half of the dopaminergic neurons of the substantia nigra has degenerated, which indicates that neurodegeneration precedes the clinical diagnosis by many years [6]. Therefore, much effort has been put into the detection of markers that indicate individuals at risk (risk markers) or indicate the neurodegenerative phase before motor symptoms allow the clinical diagnosis (prodromal markers) [5]. Risk markers such as sex, age, positive family history, genetic variances and prodromal markers like REM sleep behavior disorder, constipation, olfactory impairment or abnormal functional imaging of the dopaminergic systems have been followed in longitudinal cohort studies to determine the predictive value of single markers or marker combinations for the development of PD [7].

\section{SN hyperechogenicity as a potential premotor marker}

$\mathrm{SN}+$ has also been considered as a predictive marker, since it can be detected in about $90 \%$ of PD patients, even in the very early stages [2].
Importantly, about $10 \%$ of healthy individuals older than 50 years of age also display $\mathrm{SN}+[8]$. Considering the possibility of on-going neurodegeneration, these healthy individuals without a sign of parkinsonism but with $\mathrm{SN}+$ were further investigated in several studies, which revealed that, (i) healthy individuals with $\mathrm{SN}+$ may have nigrostriatal impairment detected by reduced presynaptic dopaminergic tracer uptake $[9,10]$, (ii) show sub-clinical motor slowing $[11,12]$, (iii) perform slightly worse in cognitive or olfaction tests $[13,14]$ compared to individuals with normal SN echogenicity and (iv) are more prone to develop severe extrapyramidal symptoms after disposure to neuroleptic drugs [15]. Furthermore, healthy individuals with increased risk for $\mathrm{PD}$, such as positive family history, asymptomatic carriers of mutations associated with PD [16,17] or REM sleep behavior disorder [18,19] also exhibited a higher rate of SN + compared to the ones without these risk and prodromal markers. All these findings in non-PD individuals support the hypothesis that $\mathrm{SN}+$ may be present before clinical diagnosis. Results of the histological analyses in postmortem brains of healthy individuals with $\mathrm{SN}+$ demonstrated an association between the size of the area of increased echogenicity and the amount of iron and microglia activity in the substantia nigra $[9,20,21]$, histological features known to be present in $\mathrm{PD}$, which gave further indication that $\mathrm{SN}+$ may reflect, or at least be related to an on-going neurodegenerative process.

To define the possible role and the strength of $\mathrm{SN}+$ in the prediagnostic stage of neurodegeneration, longitudinal studies were initiated. In the "Prospective validation of risk markers for the development of idiopathic Parkinson's disease" (PRIPS) study, 1847 healthy individuals older than 50 years were assessed biennially for the presence of $\mathrm{SN}+$ and other markers known to constitute a risk for future PD [14]. After three years of follow up, it was found that 8 out of 10 individuals with incident $\mathrm{PD}$, had $\mathrm{SN}+$ at baseline assessment, indicating a 17 -fold increased risk for PD for the participants in this range with $\mathrm{SN}+$ after three years [22]. Extension of the follow-up period to five years increased the relative risk to 20 [23]. This longitudinal study proved that $\mathrm{SN}+$ is indeed a premotor marker for PD. 


\section{Stability of SN hyperechogenicity as risk marker for prodromal PD}

So far, most studies indicate that the size of the increased echogenicity does not change over the course of the disease [24], is not related to disease severity [25], disease progression or dopamine transporter uptake [26]. Therefore, it has been categorized as a risk marker. This classification is further supported by the fact that $\mathrm{SN}+$ can also be found in very young ages unlikely to have neurodegeneration [27]. However, although the majority of the published data describe $\mathrm{SN}+$ as a stable marker, some more recent studies report correlation with disease severity or transporter uptake $[28,29]$. This may be due to a better resolution of modern ultrasound devices and thus more accurate measurements. For this reason, it is not entirely clear, whether $\mathrm{SN}+$ will be classified as risk or prodromal marker in the future.

\section{The role of SN hyperechogenicity in the diagnosis of prodromal PD}

In 2013, recommendations for the diagnosis of PD were published by the European Federation of Neurological Societies (EFNS) and Movement Disorders Society (MDS). In this EFNS/MDS guideline, TCS was recommended as an effective tool for the detection of individuals at risk for PD as well as early and differential diagnosis of PD with a high recommendation level (Level A) [30]. Further in 2015, the MDS Task Force on the definition of PD, published research criteria for the detection of prodromal PD [7]. The Task Force defined a likelihood ratio (LR) for each risk and prodromal marker according to their predictive value derived from prospective studies, and described a method based on Bayesian statistics to calculate the future probability for PD using these LRs. Among the risk and prodromal markers, presence of $\mathrm{SN}+$ is described as a strong risk marker with a positive likelihood ratio of 4.7 , which affects the calculation of the probability score more than many of the other markers [7].

\section{Limitations of $\mathrm{SN}$ hyperechogenicity for PD diagnosis}

Although considered as a strong risk marker, $\mathrm{SN}+$ should always be evaluated together with other markers as recommended in the MDS criteria. As already mentioned, about $10 \%$ of healthy individuals display $\mathrm{SN}+$, but the prevalence of $\mathrm{PD}$ is $1-2 \%$ in the elderly population, which indicates that about $80 \%$ of the healthy individuals with $\mathrm{SN}+$ will never be diagnosed with PD in their lifetime. Moreover, presence of $\mathrm{SN}+$ can also be found in other types of parkinsonism such as corticobasal degeneration or Wilson's disease [8], suggesting that additional TCS markers such as basal ganglia hyperechogenicity and size of the ventricular system are also required for the differential diagnosis. Furthermore, skill and experience are necessary to perform the assessment adequately. And finally, in around $10-15 \%$ of the population the echogenicity of the SN cannot be assessed due to the lack of bone window which prevents passing of the ultrasound waves through the skull, and limits the usage of TCS [8].

\section{Conclusion}

Today, $\mathrm{SN}+$ is recognized as a risk marker for future PD. However, the presence of $\mathrm{SN}+$ should only be taken into account in combination with other risk and prodromal markers, as indicated by the MDS research criteria for prodromal PD.

\section{References}

1. Becker G, Seufert J, Bogdahn U, Reichmann H, Reiners K (1995) Degeneration of substantia nigra in chronic Parkinson's disease visualized by transcranial color-coded real-time sonography. Neurology 45: 182-184.

2. Gaenslen A, Unmuth B, Godau J, Liepelt I, Di Santo A, et al. (2008) The specificity and sensitivity of transcranial ultrasound in the differential diagnosis of Parkinson's disease: A prospective blinded study. Lancet Neurol 7: 417-424.

3. Mahlknecht P, Seppi K, Stockner H, Nocker M, Scherfler C, et al. (2013) Substantia nigra hyperechogenicity as a marker for Parkinson's disease: A population-based study. Neurodegener Dis 12: 212-218.

4. Prestel J, Schweitzer KJ, Hofer A, Gasser T, Berg D (2006) Predictive value of transcranial sonography in the diagnosis of Parkinson's disease. Mov Disord 21: 1763-1765.

5. Postuma RB1, Aarsland D, Barone P, Burn DJ, Hawkes CH, et al. (2012) Identifying prodromal Parkinson's disease: Pre-motor disorders in Parkinson's disease. Mov Disord 27: 617-626.

6. Fearnley JM, Lees AJ (1991) Ageing and Parkinson's disease: Substantia nigra regional selectivity. Brain 114: 2283-2301.

7. Berg D, Postuma RB, Adler CH, Bloem BR, Chan P, et al. (2015) MDS research criteria for prodromal Parkinson's disease. Mov Disord 30: 1600-1611.

8. Walter U, Školoudík D (2014) Transcranial sonography (TCS) of brain parenchyma in movement disorders: Quality standards, diagnostic applications and novel technologies. Ultraschall Med 35: 322-331.

9. Berg D, Roggendorf W, Schröder U, Klein R, Tatschner T, et al. (2002) Echogenicity of the substantia nigra: Association with increased iron content and marker for susceptibility to nigrostriatal injury. Arch Neurol 59: 999-1005.

10. Walter U, Klein C, Hilker R, Benecke R, Pramstaller PP, et al. (2004) Brain parenchyma sonography detects preclinical parkinsonism. Mov Disord 19: $1445-1449$.

11. Ruprecht-Dörfler P, Klotz P, Becker G, Berg D (2007) Substantia nigra hyperechogenicity correlates with subtle motor dysfunction in tap dancers. Parkinsonism Relat Disord 13: 362-364.

12. Berg D, Seppi K, Liepelt I, Schweitzer K, Wollenweber F, et al. (2010) Enlarged hyperechogenic substantia nigra is related to motor performance and olfaction in the elderly. Mov Disord 25: 1464-1469.

13. Yilmaz R, Behnke S, Liepelt-Scarfone I, Roeben B (2016) Substantia nigra hyperechogenicity is related to decline in verbal memory in healthy elderly adults. Eur J Neurol 23: 973-978.

14. Liepelt I, Behnke S, Schweitzer K, Wolf B, Godau J, et al. (2011) Premotor signs of PD are related to SN hyperechogenicity assessed by TCS in an elderly population. Neurobiol Aging 32: 1599-1606.

15. Berg D, Jabs B, Merschdorf U, Beckmann H, Becker G (2001) Echogenicity of substantia nigra determined by transcranial ultrasound correlates with severity of parkinsonian symptoms induced by neuroleptic therapy. Biol Psychiatry 50: 463-467.

16. Schweitzer KJ, Behnke S, Liepelt I, Wolf B, Grosser C, et al. (2007) Crosssectional study discloses a positive family history for Parkinson's disease and male gender as epidemiological risk factors for substantia nigra hyperechogenicity. J Neural Transm 114: 1167-1171.

17. Brüggemann N, Hagenah J, Stanley K, Klein C, Wang C, et al. (2011) Substantia nigra hyperechogenicity with LRRK2 G2019S mutations. Mov Disord 26: 885-888.

18. Stockner H, Iranzo A, Seppi K, Serradell M, Gschliesser V, et al. (2009) Midbrain hyperechogenicity in idiopathic REM sleep behavior disorder. Mov Disord 24: 1906-1909.

19. Shin HY, Joo EY, Kim ST, Dhong H-J, Cho JW (2013) Comparison study of olfactory function and substantia nigra hyperechogenicity in idiopathic REM sleep behavior disorder, Parkinson's disease and normal control. Neurol Sci 34: 935-940.

20. Zecca L, Berg D, Arzberger T, Ruprecht P, Rausch WD, et al. (2005) In vivo detection of iron and neuromelanin by transcranial sonography: A 
Citation: Yilmaz R, Berg D (2017) Substantia Nigra Hyperechogenicity for the Early Detection of Parkinson's Disease. J Neurol Neurophysiol 8: 425. doi:10.4172/2155-9562.1000425

Page 3 of 3

new approach for early detection of substantia nigra damage. Mov Disord 20: $1278-1285$.

21. Berg D, Godau J, Riederer P, Gerlach M, Arzberger T (2010) Microglia activation is related to substantia nigra echogenicity. J Neural Transm (Vienna) 117: 1287-1292.

22. Berg D, Seppi K, Behnke S, Liepelt I, Schweitzer K, et al. (2011) Enlarged substantia nigra hyperechogenicity and risk for Parkinson disease: A 37 month 3-center study of 1847 older persons. Arch Neurol 68: 932-937.

23. Berg D, Behnke S, Seppi K, Godau J, Lerche S, et al. (2013) Enlarged hyperechogenic substantia nigra as a risk marker for Parkinson's disease. Mov Disord 28: 216-219.

24. Berg D, Merz B, Reiners K, Naumann M, Becker G (2005) Five-year follow-up study of hyperechogenicity of the substantia nigra in Parkinson's disease. Mov Disord 20: 383-385.

25. Jesus-Ribeiro J, Sargento-Freitas J, Sousa M, Silva F, Freire A, Januário C (2016) Substantia nigra hyperechogenicity does not correlate with motor features in Parkinson's disease. J Neurol Sci 364: 9-11.
26. Li DH, Zhang LY, Hu YY, Jiang XF, Zhou HY, et al. (2015) Transcranial sonography of the substantia nigra and its correlation with DAT-SPECT in the diagnosis of Parkinson's disease. Parkinsonism Relat Disord 21: 923-928.

27. Iova A, Garmashov A, Androuchtchenko N, Kehrer M, Berg D, et al. (2004) Postnatal decrease in substantia nigra echogenicity. Implications for the pathogenesis of Parkinson's disease. J Neurol 251: 1451-1454.

28. Tsai CF, Wu RM, Huang YW, Chen LL, Yip PK, et al. (2007) Transcranial color-coded sonography helps differentiation between idiopathic Parkinson's disease and vascular parkinsonism. J Neurol 254: 501-507.

29. Sanzaro E, Iemolo F (2016) Transcranial sonography in movement disorders: an interesting tool for diagnostic perspectives. Neurol Sci 37: 373-376.

30. Berardelli A, Wenning GK, Antonini A, Berg D, Bloem BR, et al. (2013) EFNS/MDS-ES/ENS [corrected] recommendations for the diagnosis of Parkinson's disease. Eur J Neurol 20: 16-34. 